\title{
3D Structural Model and Visualization of Blood Vessels Based on L-System
}

\author{
Siriprapa Ritraksa and Khamron Mekchay* \\ Department of Mathematics and Computer Science, Faculty of Science, Chulalongkorn University, \\ Bangkok 10330, Thailand
}

('Corresponding author's e-mail: khamron.m@chula.ac.th)

Received: 3 March 2021, Revised: 25 July 2021, Accepted: 1 August 2021

\begin{abstract}
The insight in structures of the blood vessels is a basis for study of blood flows to help understanding the abnormalities of blood vessels that can cause vascular diseases. Basic concept used for constructing structures of blood vessels in organs is arterial branching, which is usually characterized by fractal similarity in the bifurcation pattern. In this work, the concept of Lindenmayer system (L-system) is modified for three-dimensional (3D) tree-like structures to model structures of blood vessels in organs, and then, applied to construct and visualize structural blood vessels via our software created based on openGL and Lazarus program. The structure of blood vessels is constructed based on the physiological law of arterial branching proposed Murray (Murray's law) under additional assumptions and constraints such as the spreading of blood vessels to cover all directions, the angle condition and the non-overlapping vessels condition. The concept is applied to simulate structures of blood vessels in 3 study cases, including symmetric arterial branching, non-symmetric arterial branching and structure of blood vessel on different domains. The results of structures of blood vessels generated from all cases are measured based on the number of segments, the total blood volume and the fractal dimension. The results of modeling and simulation in this work are illustrated by comparing with other results appeared literature. Moreover, the constructed structures of the blood vessels based on this 3D L-system could be useful for future research such as blood flow, pressure and other properties involving in structures of blood vessels in different organs of human and animals.
\end{abstract}

Keywords: Lindenmayer system (L-system), Structural blood vessel, Arterial branching, Murray’s law

\section{Introduction}

The basic structure of blood vessels in human and animal organ usually has fractal pattern where each organ may have different branching structure due to the physiological law of arterial branching. The knowledge of structures of the blood vessels will be useful for study of blood flow to understand the abnormalities of blood vessels that may cause vascular diseases. Many results have been developed to describe the structures of blood vessels.

Murray [1] studied the physiological principle of minimum work applied to the angle of arterial branching to obtain angle for a simple bifurcation of blood vessels, known as Murray's law. Some model structures or network of blood vessels have been studied in human [2-4] in organ such as retina [5], heart [6-7], lung [8] and renal [9] in animal such as rat [10,30]. The structures of the blood vessels are usually used for study other topics such as blood flow in the structures [26], blood pressure in each section of blood vessels [27], and to check the position of atherosclerotic coronary arteries [28], etc. Some results of construction of structures of blood vessels involves some optimization techniques such as constrained constructive optimization (CCO) [11-13,26-27,31], volume [14-15], area [16] and flow [17,26], and some constructed structures were generated real data from images [19] and used image processing technique [30]. Most of these structure of blood vessels were constructed without using the tree-like structure of Lsystem.

L-system is basic tool for generating tree-like structures such as structure branching and root of tree, structure of blood vessels. Zamir [18] introduced parametric L-system to generate branching tree structures incorporated with the physiological laws of arterial branching. Galarreta-Valverde et al. [19] extended the traditional L-system to generate synthetic 3D blood vessels by adding stochastic rules and 
parameters. The usage of L-system in construction of tree-like structures has advantage in term of computation storage and application, where the constructed structures are stored as L-system codes instead of images.

Based on the idea of 2D L-system introduced by Zamir's work [18], in this work we modify the existing 2D and 3D L-systems in order to construct and visualize 3D structures of blood vessels. The 3D L-system is modified by using directional vectors instead of angles as first introduce in the work of Galarreta-Valverde et al. [19] to handle directions of vessels, which is much easier than using angles and rotations. In addition, the structural of blood vessels is constructed based on the physiological laws of arterial branching (Murray's law) described by Zamir [18]; Zamir and Brown [20], and with additional assumption and constraints, such as, the expanding of vessels should be in all directions to cover most region in organs, the angle constraint and the non-overlapping constraint. Moreover, we create a software based on openGL and Lazarus program to visualize and generate L-system codes of constructed structures of blood vessels.

To demonstrate the proposed construction of structures of blood vessels, we consider modelling and simulation the structures of blood vessels in 3 different sceneries:

1) Structures of blood vessels with symmetric arterial branching in a rectangular box domain.

2) Structures of blood vessels with non-symmetric arterial branching with Gaussian distribution asymmetric ratio in a rectangular box domain.

3) Structures of blood vessels in different domains ( $\mathrm{L}$ and $\mathrm{T}$ shapes).

Finally, to evaluate the performance of the constructed structures of blood vessels, we consider measurements based on the number of vessels, the total blood volume and the fractal dimension of the structures. The results of constructed structures of blood vessels are also illustrated by comparing with real images and other results appeared literature. Some discussion related to our results is also mentioned.

The organization of this work is given as follows. In section 2, we describe the methodology used for constructing structure of blood vessels. The 3 cases of modeling and simulations based on the methodology are described with results in section 3. The discussion and conclusion are given in sections 4 and 5 , respectively.

\section{Methodology}

\section{Arterial branching}

Zamir [18]; Zamir and Brown [20] described the basic structural unit of tree-like structures as an arterial bifurcation, whereby an arterial segment divides into 2 branches, and then each of the branches goes on to do the same. Assume that an arterial segment has diameter and length denoted by $d$ and $l$, respectively, with subscripts 0,1 and 2 are used to identify the parent segment and its 2 children branches, respectively, (Figure 1(a)). The basic properties at that bifurcation for $d_{0}, d_{1}, d_{2}$ and $l_{0}, l_{1}, l_{2}$ together with the angles $\theta_{1}$ and $\theta_{2}$ of the 2 children branches making with the parent are shown in Figure 1(a), where the subscript 1 is for the branch with larger value.

The symmetry of branching is defined using the bifurcation index or asymmetry ratio $\alpha=\frac{d_{2}}{d_{1}}$, which has the value in the range of $0-1.0 ; \alpha=1.0$ when the 2 diameters are equal or symmetric and $\alpha \approx 0$ when one of the 2 diameters is very much larger than the other. According to Murray's law [1], also known as cube law or power law (with $\mathrm{k}=3$ ), the relations between the diameters and the lengths of the 3 vessels at the branching can be defined in terms of $\alpha$ as:

$\frac{d_{1}}{d_{0}}=\frac{l_{1}}{l_{0}}=\frac{1}{\left(1+\alpha^{3}\right)^{1 / 3}}, \frac{d_{2}}{d_{0}}=\frac{l_{2}}{l_{0}}=\frac{\alpha}{\left(1+\alpha^{3}\right)^{1 / 3}}$.

Moreover, based on the cube law for minimizing the pumping power required to drive the blood flow, the angles $\theta_{1}$ and $\theta_{2}$ of the 2 branches making with the parent at the bifurcation can be obtained in term of index ratio from the relations:

$\cos \theta_{1}=\frac{\left(1+\alpha^{3}\right)^{4 / 3}+1-\alpha^{4}}{2\left(1+\alpha^{3}\right)^{2 / 3}}, \cos \theta_{2}=\frac{\left(1+\alpha^{3}\right)^{4 / 3}+\alpha^{4}-1}{2 \alpha^{2}\left(1+\alpha^{3}\right)^{2 / 3}}$.

\section{L-systems}

Originally, L-system was conceived as a mathematical theory for simple plant development which did not have enough detail for comprehensive modeling of higher plants [21]. Several geometric interpretations of L-systems were proposed to turn them into versatile tools for plant modeling. A general 
2D L-system code consists of symbols such as direction $(+,-)$, diameter (D), angle $(\theta)$, and length (L); for example, $\mathrm{F}\left(l_{0}, d_{0}\right)\left[-\left(\theta_{1}\right) \mathrm{F}\left(l_{1}, d_{1}\right)\right]\left[+\left(\theta_{2}\right) \mathrm{F}\left(l_{2}, d_{2}\right)\right]$ is corresponding to a picture shown in Figure 1(a). The meanings of symbols based on Prusinkiewicz and Lindenmayer [22] are as follows:

$\mathrm{L}$ is length of a line.

$\mathrm{D}$ is diameter of a line.

$\mathrm{F}(\mathrm{L}, \mathrm{D})$ is draw a line forward with length $\mathrm{L}$ and diameter $\mathrm{D}$.

$+(\theta)$ is turn left by angle $\theta$.

$-(\theta)$ is turn right by angle $\theta$.

[ is push the current state onto a push down operations stack.

] is pop a state from the stack and make it the current state; no line is drawn.

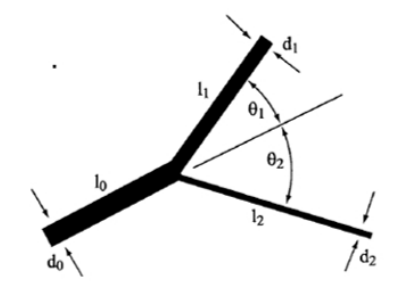

$\mathrm{F}\left(l_{0}, d_{0}\right)\left[-\left(\theta_{1}\right) \mathrm{F}\left(l_{1}, d_{1}\right)\right]\left[+\left(\theta_{2}\right) \mathrm{F}\left(l_{2}, d_{2}\right)\right]$

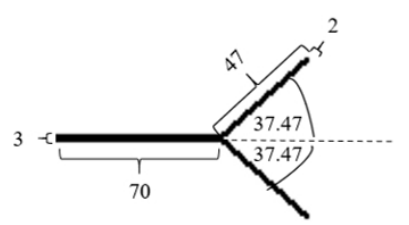

$F(70,3)[-(37.47) F(47,2)][+(37.47) F(47,2)]$

(a)

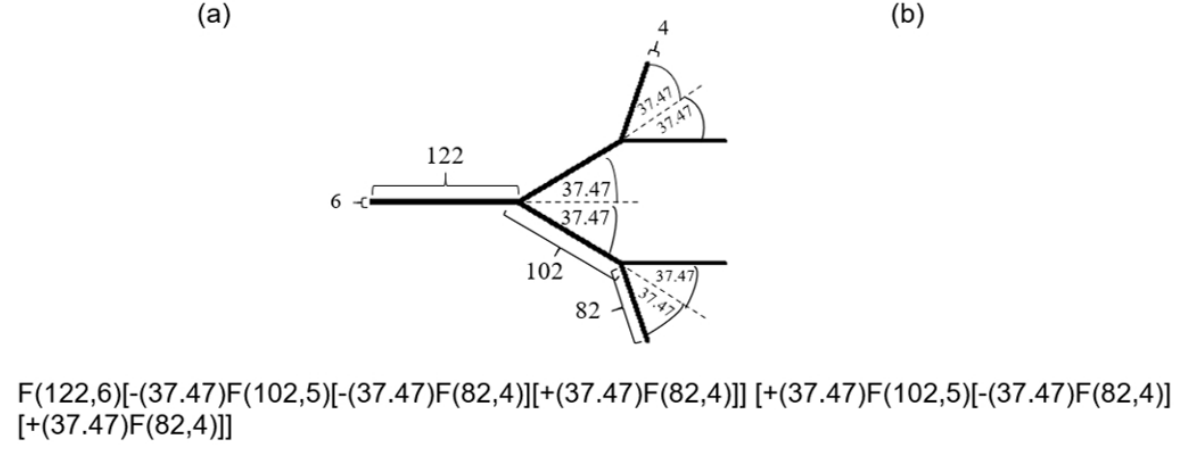

(b)

(c)

Figure 1 (a) The basic variables at an arterial bifurcation [18,20]. Examples of L-system codes and corresponding images: (b) 1 generation and (c) 2 generations.

Examples of L-system codes and the corresponding generated images are shown in Figures 1(b) and 1(c). In the code of Figure 1(b), the line is drawn with length 70 and diameter 3; then the $1^{\text {st }}$ branch is a line with length 47 and diameter 2 to the right turn with angle $37.47^{\circ}$, and the $2^{\text {nd }}$ branch is a line with length 47 and diameter 2 to the left turn with angle $37.47^{\circ}$. Figure 1(c) shows the code and its corresponding image for 2-generations of branching.

In this work, the concept of 2D L-system introduced by Zamir [18] for generating tree-like structures is modified to handle 3D structures of blood vessels, instead of using existing 3D L-system that is quite complicated to apply, as described in the next section.

\section{Construction structural blood vessel}

Modification of 3D L-system

Based on the general concept of 2D L-system, we modify the idea to get a new 3D L-system by using directional vectors to indicate the directions of new branches instead of using the $3 \mathrm{D}$ L-system proposed by Prusinkiewicz et al. [23], which used rotation angles on 3 axes: $+(\theta),-(\theta)$ for turning left and right by angle $\theta$ around the $\mathrm{U}$ axis, respectively; $\&(\theta), \wedge(\theta)$ for pitching down and up by angle $\theta$ around the $\mathrm{L}$ axis, respectively; and $/(\theta), \backslash(\theta)$ for rolling left and right by angle $\theta$ around the $\mathrm{H}$ axis, respectively (Figure 2(a)). Since using rotations on 3 axes is complicated for constructing 3D structure of blood vessels based on our proposed model, the 3 angles are replaced by 1-unit directional vector for the direction of a new branches. This modification makes the construction of L-system codes and 
visualization of structures of blood vessels simple to handle. The modified 3D L-system code is generated as text commands similar to other existing L-systems.

An example of this 3D L-system code is $F(L, D)[-(x, y, z) F(L, D)][+(x, y, z) F(L, D)]$, where the meanings of symbols are given as follows:

$\mathrm{L}$ is length of a line.

$\mathrm{D}$ is diameter of a line.

$\mathrm{F}(\mathrm{L}, \mathrm{D})$ is draw a line forward with length $\mathrm{L}$ and diameter $\mathrm{D}$.

$(\mathrm{x}, \mathrm{y}, \mathrm{z})$ is unit directional vector.

$-(\mathrm{x}, \mathrm{y}, \mathrm{z})$ is $1^{\mathrm{st}}$ branch moving in the direction $(\mathrm{x}, \mathrm{y}, \mathrm{z})$.

$+(\mathrm{x}, \mathrm{y}, \mathrm{z})$ is $2^{\text {nd }}$ branch moving in the direction $(\mathrm{x}, \mathrm{y}, \mathrm{z})$.

[ is push the current state onto a push down operations stack.

] is pop a state from the stack and make it the current state; no line is drawn.

Examples of 3D L-system codes and their corresponding generated images are shown in Figures 2(b) and 2(c). Figure 2(b) shows the image for the code $F(602,64)[-(0.79,-0.51,0.32) F(478,51)]$ $[+(0.79,0.49,-0.35) \quad F(478,51)]: \quad F(602,64)$ draws a line with length 602 and diameter 64; $[-(0.79,-0.51,0.32) \mathrm{F}(478,51)]$ construct the $1^{\text {st }}$ line with length 478 and diameter 51 in direction $(0.79$,$0.51,0.32)$; and $[+(0.79,0.49,-0.35) \mathrm{F}(478,51)]$ construct the $2^{\text {nd }}$ line with length 478 and diameter 51 in direction $(0.79,0.49,-0.35)$. Figure 2(c) shows the image of 2 generations of branching corresponding to the L-system code $\mathrm{F}(396,49)[-(0.7,0.4,0.3) \quad \mathrm{F}(314,39) \quad[-(0.2,0.7,0.5) \quad \mathrm{F}(250,31)][+(0.6,0.7,-0.2)$ $\mathrm{F}(250,31)]][+(0.7,-0.4,-0.3) \mathrm{F}(314,39)[-(0.7,-0.5,0.2) \mathrm{F}(250,31)][+(0.2,-0.8,-0.3) \mathrm{F}(250,31)]]$.

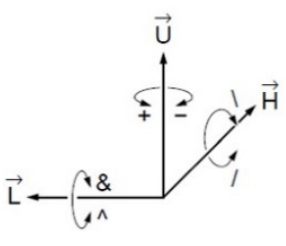

(a)

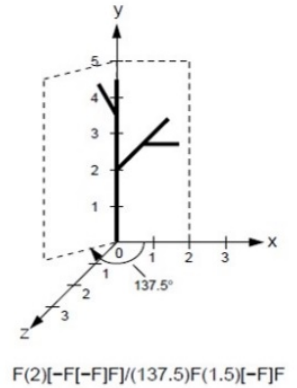

$F(2)[-F[-F] F] /(137.5) F(1.5)[-F] F$

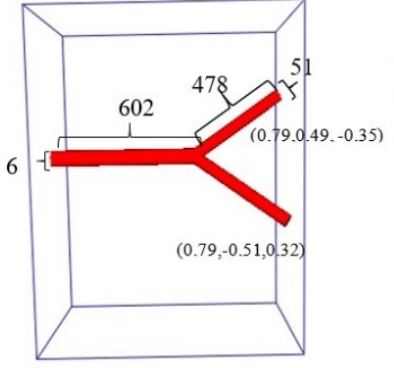

(b)

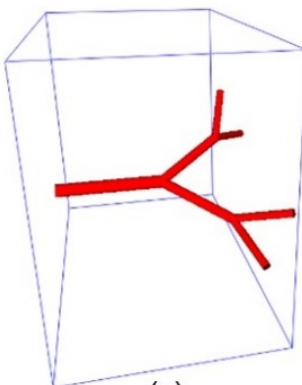

(c)

Figure 2 (a) 3D L-system with angles purpose by Prusinkiewicz et al. [23]. Examples of images from modified 3D L-system: (b) 1 generation and (c) 2 generations.

\section{Construction of L-system code for a structure of blood vessels}

The steps for generating L-system code for the construction of 3D structural blood vessels are given in the Algorithm I as the followings.

\section{Algorithm I (Construction of L-system for structure of blood vessels)}

1) Input initial parameters.

a) For a rectangular box domain, we input the dimension as $L, W, H$ for the length, the width, and the height, respectively.

b) Starting point of blood vessels.

c) The total number of generations for the structure of blood vessels, $n$.

d) The initial asymmetric ratio $\alpha_{0}$.

2) Calculate the initial length and diameter of parent vessel.

a) Calculate initial blood volume.

To calculate the initial volume, we assume that the blood from parent vessel transfers completely to the 2 children branches at each arterial bifurcation. In addition, based on result in literature, $7 \%$ of the average blood volume in body weight [25] is used for calculating the initial volume in $1^{\text {st }}$ bifurcation $\left(1^{\text {st }}\right.$ generation) according to the formula: 
$V_{0}=\frac{(W \times L \times H)}{n} \times k$

where $W, L$ and $H$ are the dimension of the domain, $n$ is the number of generations, and $k$ is constant assumed to be $7 \%$.

b) Compute initial length.

We assume the combined length of all larger vessels is proportional to the length of the domain. In this study we assume that they are equal, and we can calculate the initial length from the relation:

$L=L_{0}+L_{1}+\ldots+L_{n}$

where $L_{1}=\frac{L_{0}}{\left(1+\alpha^{3}\right)^{1 / 3}}, L_{2}=\frac{L_{1}}{\left(1+\alpha^{3}\right)^{1 / 3}}, \ldots, L_{n}=\frac{L_{n-1}}{\left(1+\alpha^{3}\right)^{1 / 3}}=\frac{L_{0}}{\left(1+\alpha^{3}\right)^{n / 3}}$, when $L_{i}$ denotes the length of larger vessel created in $i$-th generation. Thus,

$L=L_{0}+\frac{L_{0}}{\left(1+\alpha^{3}\right)^{1 / 3}}+\cdots+\frac{L_{0}}{\left(1+\alpha^{3}\right)^{n / 3}}=L_{0}\left[1+\frac{1}{\left(1+\alpha^{3}\right)^{1 / 3}}+\cdots+\frac{1}{\left(1+\alpha^{3}\right)^{n / 3}}\right]$,

therefore, $L_{0}=\frac{L}{\left[1+\frac{1}{\left(1+\alpha^{3}\right)^{1 / 3}}+\cdots+\frac{1}{\left(1+\alpha^{3}\right)^{n / 3}}\right]}=\frac{L}{S_{n}}$,

where $S_{n}=\sum_{k=0}^{n} r^{k}=\frac{1-r^{n+1}}{1-r}$ with $\quad r=\frac{1}{\left(1+\alpha^{3}\right)^{1 / 3}}$.

c) Compute initial diameter.

From the relation $V_{0}=\frac{\pi L_{0} D_{0}^{2}}{4}$, we can calculate $D_{0}$ from $D_{0}=\sqrt{\frac{4 V_{0}}{\pi L_{0}}}$.

3) Calculate parameters of the 2 new branches.

Based on Zamir [18] (Murray's law), we calculate parameters of the 2 new branches as follow:

$l_{1}=\frac{L_{0}}{\left(1+\alpha^{3}\right)^{1 / 3}}, \quad l_{2}=\frac{\alpha L_{0}}{\left(1+\alpha^{3}\right)^{1 / 3}}$,

$d_{1}=\frac{D_{0}}{\left(1+\alpha^{3}\right)^{1 / 3}}, \quad d_{2}=\frac{\alpha D_{0}}{\left(1+\alpha^{3}\right)^{1 / 3}}$,

and $\cos \theta_{1}=\frac{\left(1+\alpha^{3}\right)^{4 / 3}+1-\alpha^{4}}{2\left(1+\alpha^{3}\right)^{2 / 3}}, \quad \cos \theta_{2}=\frac{\left(1+\alpha^{3}\right)^{4 / 3}+\alpha^{4}-1}{2 \alpha^{2}\left(1+\alpha^{3}\right)^{2 / 3}}$,

$\theta_{1}=\arccos \left(\frac{\left(1+\alpha^{3}\right)^{4 / 3}+1-\alpha^{4}}{2\left(1+\alpha^{3}\right)^{2 / 3}}\right), \theta_{2}=\arccos \left(\frac{\left(1+\alpha^{3}\right)^{4 / 3}+\alpha^{4}-1}{2 \alpha^{2}\left(1+\alpha^{3}\right)^{2 / 3}}\right)$.

4) Calculate the appropriate directions of 2 branches.

To obtain appropriate adjusted direction in this step, we denote the followings. Let $\overrightarrow{P_{1}}$ be the direction of the parent vessel from the current position with unit vector $\widehat{P}_{1} ; \overrightarrow{P_{2}^{1}}$ be the selected direction for the larger branch obtained from the construction model and $\widehat{P_{v}^{1}}$ be the appropriate adjusted direction satisfying to have angle $\theta_{1}$ with the parent direction, Figure 3. Note that, the selected direction $\overrightarrow{P_{2}^{1}}$ is obtained based on the construction constraint described in the next section, which might not followed the angle condition of Zamir's rule given in step 3 of Algorithm I, and we need to adjust to obtain an appropriate direction $\widehat{P_{v}^{1}}$.

To obtain $\widehat{P_{v}^{1}}$, we assume this vector lies on the plane containing both the parent direction $\overrightarrow{P_{1}}$ and the selected direction $\overrightarrow{P_{2}^{1}}$. The calculation is obtained based on basic knowledge of vector calculus as follows:

$\widehat{P_{v}^{\imath}}=\frac{\overrightarrow{P_{v}^{\imath}}}{\left\|\overrightarrow{P_{v}^{i}}\right\|}$, where $\overrightarrow{P_{v}^{\imath}}=\widehat{P}_{1}+\widehat{P}_{3}^{\imath} \tan \theta$

when $\widehat{P_{3}^{l}}=\frac{\overrightarrow{P_{3}^{l}}}{\left\|\overrightarrow{P_{3}^{l}}\right\|}, \quad \overrightarrow{P_{3}^{l}}=\frac{\overrightarrow{P_{2}^{l}}}{\overrightarrow{P_{1}} \overrightarrow{P_{2}^{l}}}-\widehat{P_{1}}$, as calculated according to Figure 3. 
After the calculation, we check the assumption constraint of $\widehat{P_{v}^{1}}$ according to construction constraints described in the next section; if it satisfies the constraints, then added $\widehat{P_{v}^{1}}$ to the L-system code for the direction of the larger branch as $\left[-\widehat{P_{v}^{1}} F\left(l_{1}, d_{1}\right)\right]$. Similarly, the same calculation is repeated for the smaller branch having selected direction $\overrightarrow{P_{2}^{2}}$ to have angle $\theta_{2}$ to obtain $\widehat{P_{v}^{2}}$ to be included in the L-system code as $\left[+\widehat{P_{v}^{2}} F\left(l_{2}, d_{2}\right)\right]$.

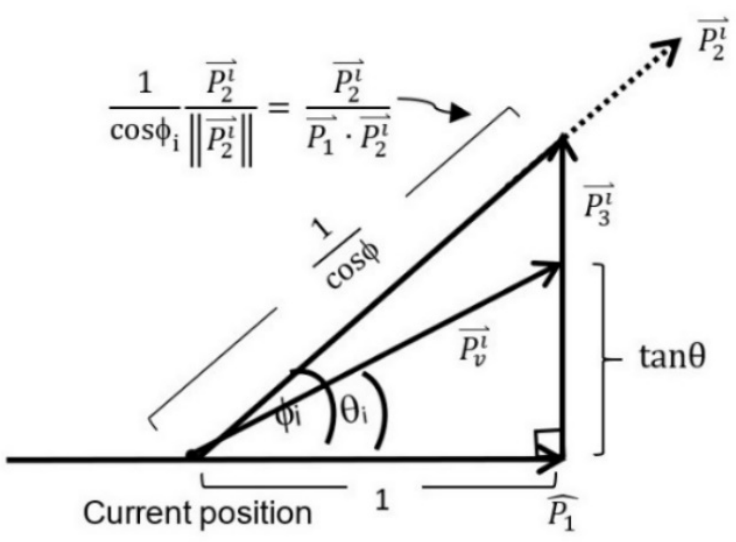

Figure 3 Diagram of directional vectors $\overrightarrow{P_{1}}, \overrightarrow{P_{2}^{l}}, \overrightarrow{P_{3}^{\imath}}$ and $\overrightarrow{P_{v}^{l}}$.

5) Update L-system code.

6) Repeat steps 3 to 5 until complete $n$ generations.

7) Output: L-system code for visualization described in the next section.

\section{Construction constrains}

As mentioned in the construction procedure described in 2.3.2, the construction of L-system file for the construction of structure of blood vessels relies on the selection of directions of new vessels from the parent as described in step 4 of Algorithm I. The selection of direction $\overrightarrow{P_{2}^{1}}$ is obtained according to the following assumption and constraints.

\section{Angle constraint}

According to angle condition for a simple bifurcation [1], the angle between the parent and a new vessel should be less than $90^{\circ}$. The angle between parents and new branch is computed from = $\cos ^{-1}\left(\widehat{P_{1}} \cdot \widehat{P_{v}^{1}}\right)$, where $\widehat{P_{1}}$ and $\widehat{P_{v}^{2}}$ are unit directional vectors of the parent and a new branch, respectively. The new branch (vessel) is considered to satisfy the angle constraint when the angle is $<90^{\circ}$.

\section{Non-overlapping vessels stay inside domain} domain.

This constraint state that the vessels do not overlap or are close to other vessels, and are inside the

1) To check that a new vessel does not overlap or is close to other vessels, we compute distances from the terminal position of the new constructed vessel to all other vessels. In this work, the new vessel is considered as non-overlapping or close to other vessels if all distances are $>70 \%$ of the length of the new vessel, otherwise it is considered as overlapped.

2) To check that a new vessel is inside the domain, we check the coordinate $(x, y, z)$ of the terminal position; the values of $x, y$ and $z$ must be in the range of the domain to be considered as inside the domain.

The vessels expand throughout the domain

The selection of directions for the 2 new vessels is decided based on the distances from the current position to the corners of domain, namely, the vessel should go in the direction of the farthest corner. In 
this case, we select the direction of the $1^{\text {st }}$ vessel to be that of the longest distance, and the direction of the next longest will be for the $2^{\text {nd }}$ vessel.

The selection of the direction of a new vessel based on above constraints is given in Algorithm II described below. In the case that an appropriate direction is not obtained due to the constraints, the new vessel will not be constructed at the bifurcation. The flow charts of both Algorithms I and II are shown in Figure 4.

Algorithm II

Let $\left(x_{c}, y_{c}, z_{c}\right)$ be the current terminal position of the parent vessel and

$\left(x_{i}, y_{i}, z_{i}\right), i=1,2, \ldots, 8$ be the coordination of 8 corners of the domain.

1) Compute distances

$d_{i}=\sqrt{\left|x_{c}-x_{i}\right|^{2}+\left|y_{c}-y_{i}\right|^{2}+\left|z_{c}-z_{i}\right|^{2}}$.

2) Arrange $d_{j_{1}} \geq d_{j_{2}} \geq \cdots \geq d_{j_{8}}$.

3 ) If the angle constraint is required in the model, then check angle constrain of $d_{j_{i}}$, $i=1,2, \ldots, 8$ :

- if $d_{j_{i}}$ satisfies angle constraint

$\circ \quad$ select $\overrightarrow{p_{2}^{1}}=\left\langle x_{j_{i}}-x_{c^{\prime}} y_{j_{i}}-y_{c^{\prime}} z_{j_{i}}-z_{c}\right\rangle$ to use for step 4 in Algorithm I.

- $\quad$ else

$\circ \quad$ take $d_{j_{i}}$ for the next $i$ and go back to step 3 . adjusted

4) Check the non-overlapping constraint of $\widehat{p_{v}^{1}}$ obtained from Algorithm I after the direction is

- $\quad$ if satisfied

$\circ$ adding $\widehat{p_{v}^{1}}$ in the L-system file.

- $\quad$ else

$\circ \quad$ take $d_{j_{i}}$ for the next $i$ and go to step 3 .

5) Select $d_{j_{i}}$ for the next $i$ and repeat steps 3 and 4 for obtaining $\overrightarrow{p_{2}^{2}}$ and $\widehat{p_{v}^{2}}$.

6) If all directions for $d_{j_{1}}$ do not satisfy constraints, then a new vessel will not be created at this bifurcation. 


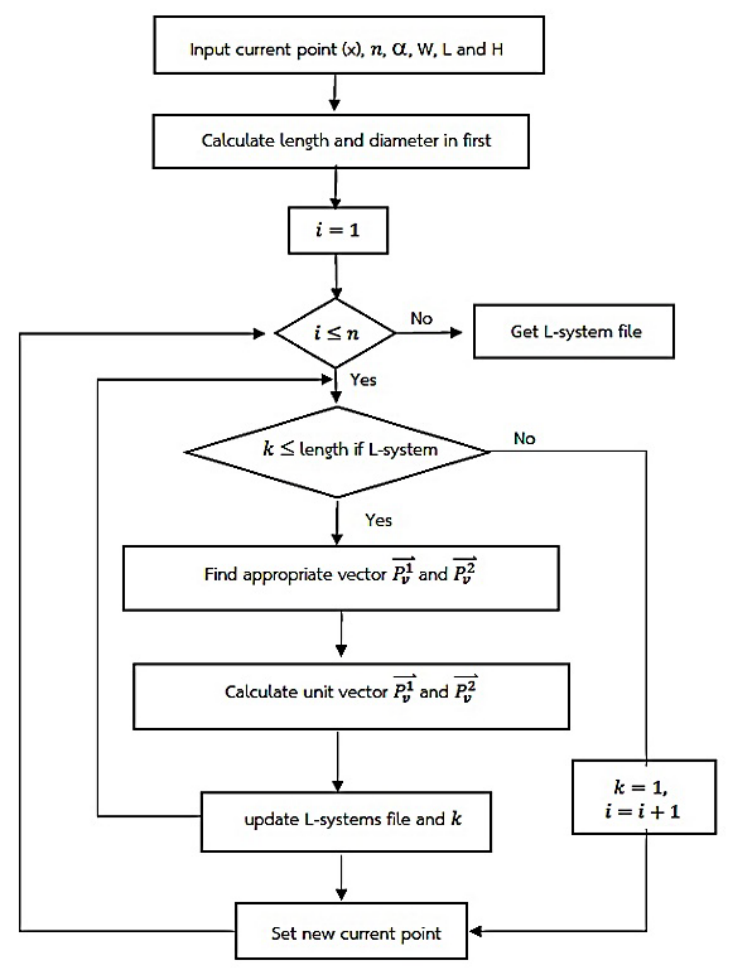

(a)

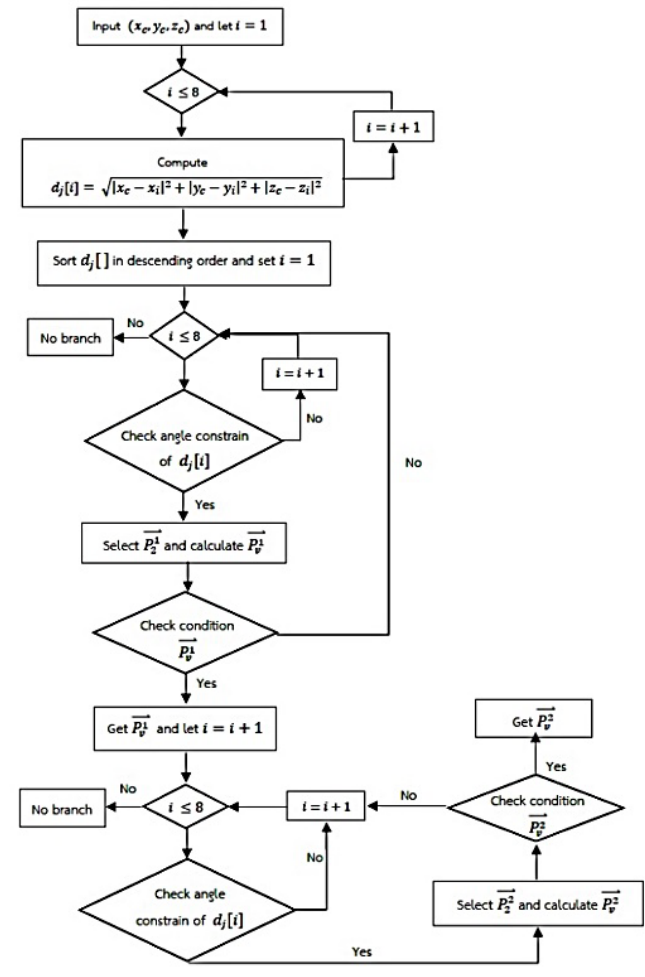

(b)

Figure 4 Flowchart of (a) Algorithm I and (b) Algorithm II.

\section{Visualization and software}

In this work, the visualization is incorporated by our created software based on openGL and Lazarus program. The feature of the program is simply shown in Figure 5. The program will perform 2 different tasks based on the construction procedures described in previous sections; 1) create an L-system code and 2) produce $3 \mathrm{D}$ visualization of structures of blood.

Figure 5 shows the main window of the program: The area in the red frame is for showing the constructed 3D structure of blood vessels; the green frame is for showing the L-system code generated from the construction; the blue frame is the menu for inputting initial parameters including some buttons for the program operation, such as open and save L-system code file with the white box showing the performance measurements. Users can run the program to construct a structure of blood vessels for both L-system code and 3D visualization by inputting initial required parameters in the blue region. The program will generate L-system code and file based on the Algorithms I and II described in the previous section. When the program finishes running, it will show a pop-up small window "L-system finished" and string of texts of L-system code in the green box. Finally, by pressing the "OK" button, the result of 3D image of a structure of blood vessels will display in the red box. The 3D visualization image can be zoomed and rotated by clicking and holding mouse. We can save the constructed L-system code as a text file format by press the "save file" button, and a window will pop up for selecting file name to save. The program can also read an already existed L-system code file to generate a 3D visualization of a structure of blood vessels. This is performed by pressing the open file button, which will pop up window to choose a file, and the result 3D image of the corresponding structure will be displayed by clicking "open file". 


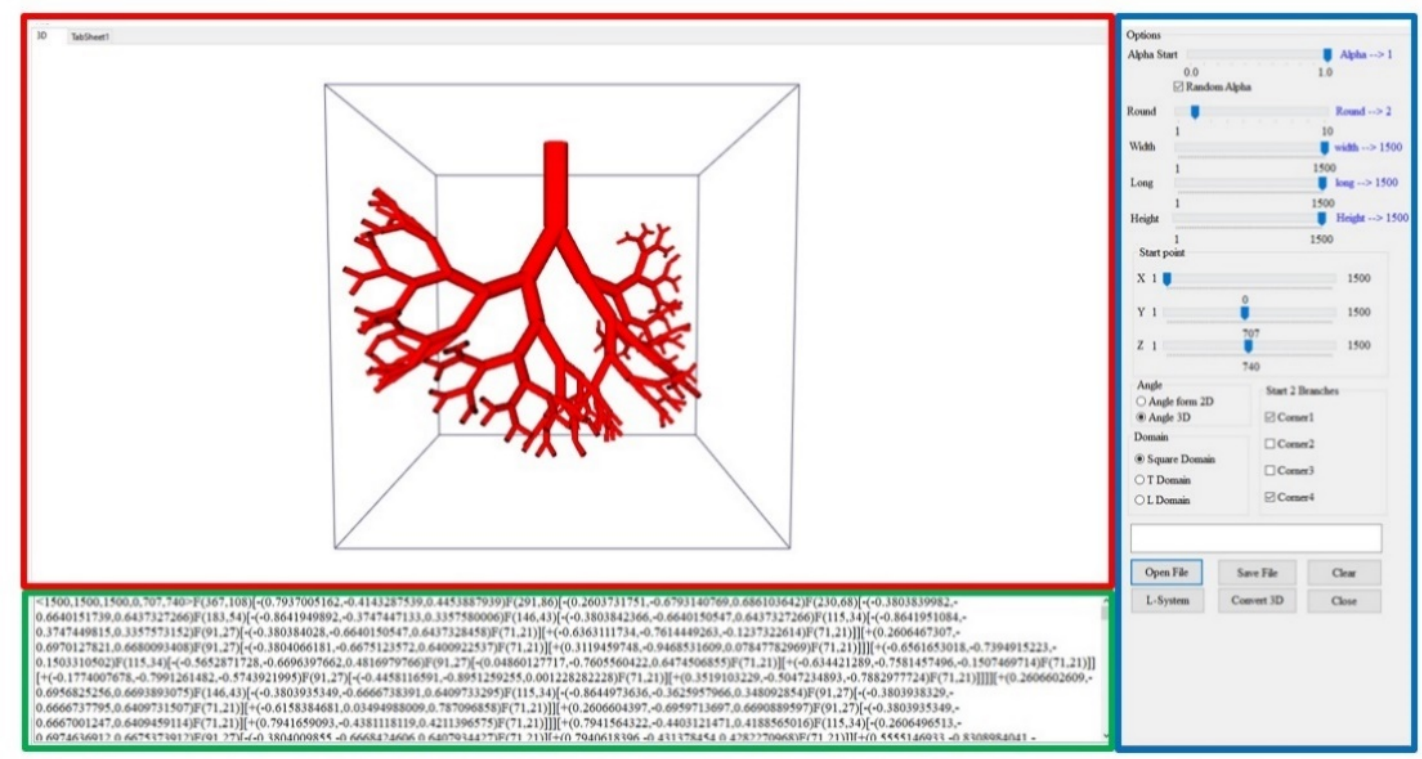

Figure 5 The window of the software for construction and visualization of a structure of blood.

The advantage of L-system file is clearly observed in term of the storage for the visualization of 3D structure of blood vessels; the storage of L-system file requires much less space than that of many images captured in many angles. The L-system code file can be transformed into 3D visualization via our implemented program, where the result can be zoomed and viewed in any angles by rotations. Another point that can be an advantage is that the L-system code file can be applied for additional study of structures of blood vessels such as blood flow, blood pressure, etc.

\section{Modeling and simulation results of structures of blood vessels}

To illustrate the construction model of structures of blood vessels of this work, we perform simulations of structures of blood vessels in 3 different sceneries.

\section{Structures of symmetric arterial branching $(\alpha=1)$}

This model studies the effect of the angle condition together with pre-selected directions of the $1^{\text {st }}$ generations on the structures of blood vessels. The construction is for rectangular box domain with constant $\alpha=1$ in all generations for symmetric branching. We divide the study into 3 cases.

Case 1: No pre-selected directions for the $1^{\text {st }}$ generation under the angle

In this case, the construction of the structures of blood vessels follows the Algorithms I and II.

\section{Case 2: Pre-selected directions for the $1^{\text {st }}$ generation under the angle constrain}

In this case, at the $1^{\text {st }}$ arterial branching, the directions are pre-selected to separate the 2 new vessels. After that, the constructions of the next generations follow Algorithms I and II similar to Case 1.

\section{Case 3: Pre-selected directions for the $1^{\text {st }}$ generation without the angle constrain}

In this case, the directions of the 2 new vessels are pre-selected similar to Case 2; however, after that, the constructions of new vessels for the next generations follows the Algorithms I and II without the angle constraint, i.e., the angle constraint is not required in the step 3 of Algorithm II.

To analyze and compare structures of blood vessels generated from these 3 cases, we measure based on the following criteria:

1) The number of segments, i.e., the number of blood vessels created in the structure.

2) The total blood volume the structure can have, which is calculated from the sum of blood volume from all vessels.

3) The fractal dimension $(\delta)$ calculated based on Yang and Wang [3]: 
$\delta_{i}=\frac{\ln 2}{\ln 2-\ln \beta_{i}}$

where, $\beta_{i}= \begin{cases}\frac{\left(d_{1 i}\right)^{2}+\left(d_{2 i}\right)^{2}}{\left(d_{0_{i}}\right)^{2}}, & \text { if it has } 2 \text { new vessels created; } \\ \frac{\left(d_{1_{i}}\right)^{2}}{\left(d_{0_{i}}\right)^{2}}, & \text { if it has only } 1 \text { vessel created, }\end{cases}$

and obtain $\delta=\frac{\sum_{i=1}^{N} \delta_{i}}{N}$, when $\mathrm{N}$ is the number of bifurcations in the structure that have at least 1 vessel created; $\beta_{i}$ is the value at the $i$-th bifurcation; $d_{0_{i}}, d_{1_{i}}$, and $d_{2 i}$ are diameters of parent vessel, and the 2 children's vessels at the $i$-th bifurcation, respectively; and $\delta_{i}$ is the index at the $i$-th bifurcation.

Note that, for structure with symmetric arterial branching, $\alpha=1$, the fractal dimension is exactly 1.5 when all bifurcations have 2 children, according to Murray's law described in section 2.1. In all cases, the fractal dimension will be less than 1.5 because some bifurcations may have only 1 vessel created, and the number will be close to 1.5 when almost all bifurcations have 2 vessels created.

The construction and the measurement values of the structures of blood vessels of the 3 cases are shown in Figure 6 when using 5, 6 and 7 generations.

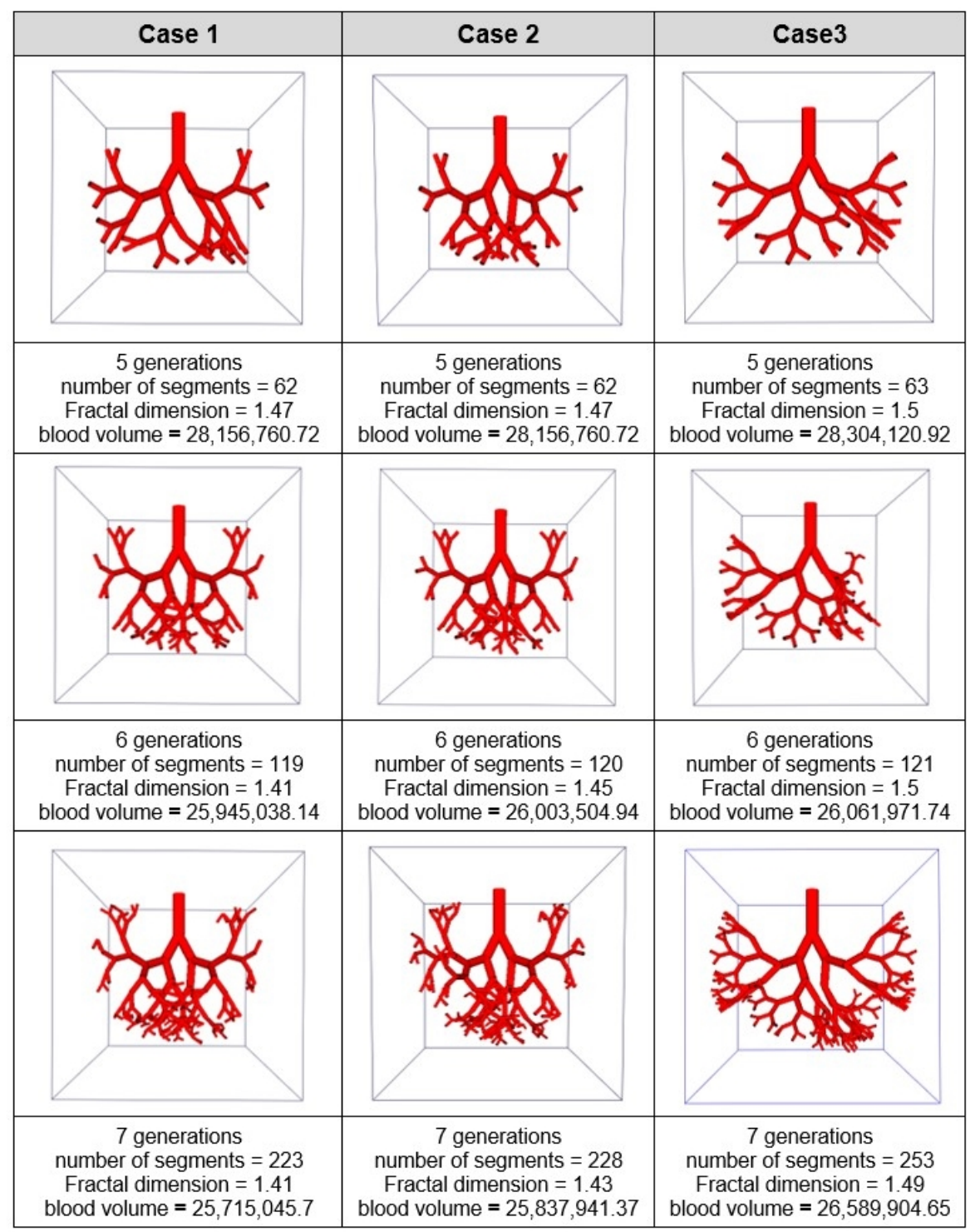

Figure 6 Structures of blood vessels for structures with symmetric arterial branching describe in 3.1. 
From the construction of structures of blood vessels for the 3 cases of section 3.1 shown in Figure 6, we have the following observations.

1) When considered using the same number of generations, the numbers of vessel segments created in the structures can be ordered as: Case $1<$ Case $2<$ Case 3 . In addition, the structure produced in Case 3 seem to have more vessels and cover more area than the other 2 cases. This implies that the structures of blood vessels has more vessels when the construction has less constraint such as in Case 3, when the angle constraint was not used for the construction.

2) When measure the structures based on the fractal dimension, we found that in this symmetric bifurcation, all 3 cases produced values close to 1.5. This suggested that the construction of structures in all 3 cases are similar in the sense that they produce structures that are almost complete. We also observed that when the numbers of generations is high, $n>7$, the fractal dimension is smaller than 1.5. This suggests that when the number of generation is high, there are many vessels already created in the structure, therefore, it might be difficult to create a new vessel due to the "non-overlapping" constraint, and a bifurcation might be incomplete.

3) Based on blood volume measurement, all 3 cases produce structures of blood vessels in the same way as that described in 1), namely, Case 3 has more blood volume than the other cases and structures with higher generations have smaller blood volume. This suggests that the blood volume follows directly with the numbers of blood vessels created in the structure, when using the same number of generations.

Structures of non-symmetric arterial branching with Gaussian distribution asymmetric ratio $\alpha$

Based on Murray's law, the non-symmetric at arterial branching is controlled by the asymmetric ratio $\alpha$, which affects the lengths, diameters, and angles at bifurcations. In this section, we apply the Case 3 of section 3.1 by using the asymmetric ratio $\alpha$ in the range $0.6-1.0$ as reported in Zamir [7] and also has Gaussian distribution, which is considered in 3 cases. Note that in this construction, the ratio $\alpha$ used in step 3 of Algorithm I varies at each bifurcation from Gaussian distribution.

Case 1: Gaussian distribution a with mean 0.9

In step 3 in Algorithm I, the $\alpha$ is generated from $\alpha=0.9+0.1 N(0,0.04)$, where $N(0,0.04)$ is a normal distribution with mean $=0$ and variance $=0.04$.

Case 2: Gaussian distribution $\alpha$ with mean 0.85

In step 3 in Algorithm I, the $\alpha$ is generated from $\alpha=0.85+0.15 N(0,0.04)$, where $N(0,0.04)$ is a normal distribution with mean $=0$ and variance $=0.04$.

Case 3: Gaussian distribution $\alpha$ with mean 0.8

In step 3 in Algorithm I, the $\alpha$ is generated from $\alpha=0.8+0.2 N(0,0.04)$, where $N(0,0.04)$ is a normal distribution with mean $=0$ and variance $=0.04$.

The construction of structures of blood vessels of these 3 cases are measured using the number of vessels, the fractal dimension, and the blood volume. The visualization and measurement values of structures of blood vessels constructed of these 3 cases are shown in Figure 7. 


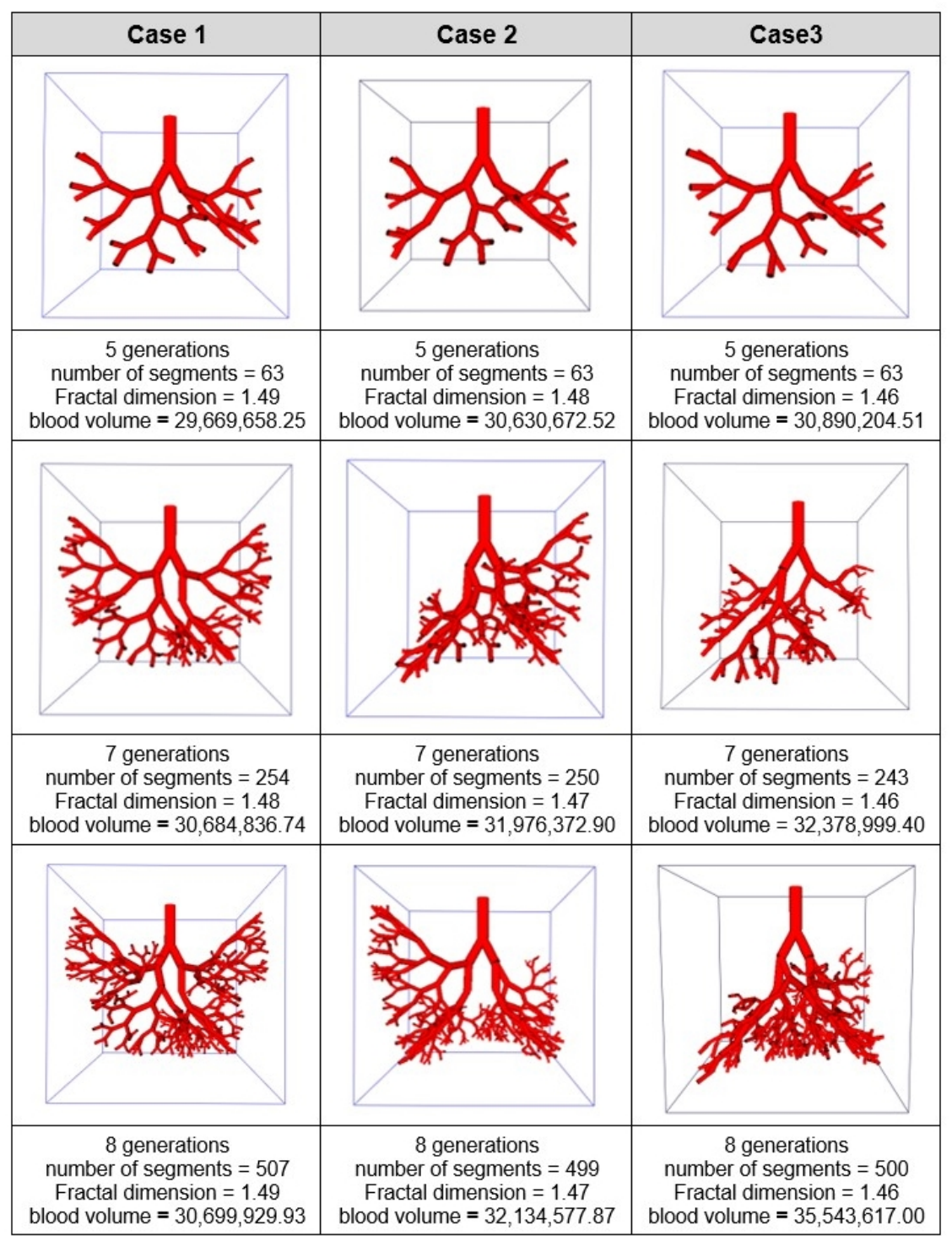

Figure 7 Structures of blood vessels for structures with non-symmetric arterial branching describe in 3.2.

As shown in Figure 7 for structures with non-symmetric arterial branching, the fractal dimension of structures produced by Case $1(\alpha \approx 0.9)$ have values closer to 1.5 than the other cases as expected, because Case 1 is more symmetric than Cases 2 and 3. However, when consider the numbers of vessels and blood volumes, the structures of blood vessels produced from these 3 cases have no significant different within the same number of generations. Due to non-symmetric ration, the images of structures of blood vessels have non-symmetric structures (Figure 7) as compared with those images for symmetric structures in section 3.1 (Figure 6).

\section{Structures constructed $L$ and $T$ shape domains}

In this section, we construct the structures of blood vessels by extending the domain from rectangular boxes to other domains such as $\mathrm{L}$ and $\mathrm{T}$ shapes. This study is to illustrate the construction Algorithms I and II, which can be applied for other type of domains by showing that the model of construction described in section 2 can be applied to generate structures of blood vessels for $\mathrm{L}$ and $\mathrm{T}$ shape domain.

The structures of blood vessels are constructed based on the Algorithms I and II with Gaussian distribution asymmetric ratio $\alpha$ with mean 0.9 and without the angle constraint. Due to the change of geometry of the domain, the algorithm is modified so that the corners of the new domain are update during the creation of new vessels. 
The construction of structures of blood vessels for L and T shape domains are shown in Figure 8. The results demonstrate that construction model can be used to simulate structures of blood vessels in different domain such as $\mathrm{L}$ and $\mathrm{T}$ shapes. As observed in Figure 8, vessels seem to move to empty space to cover area of the domain.

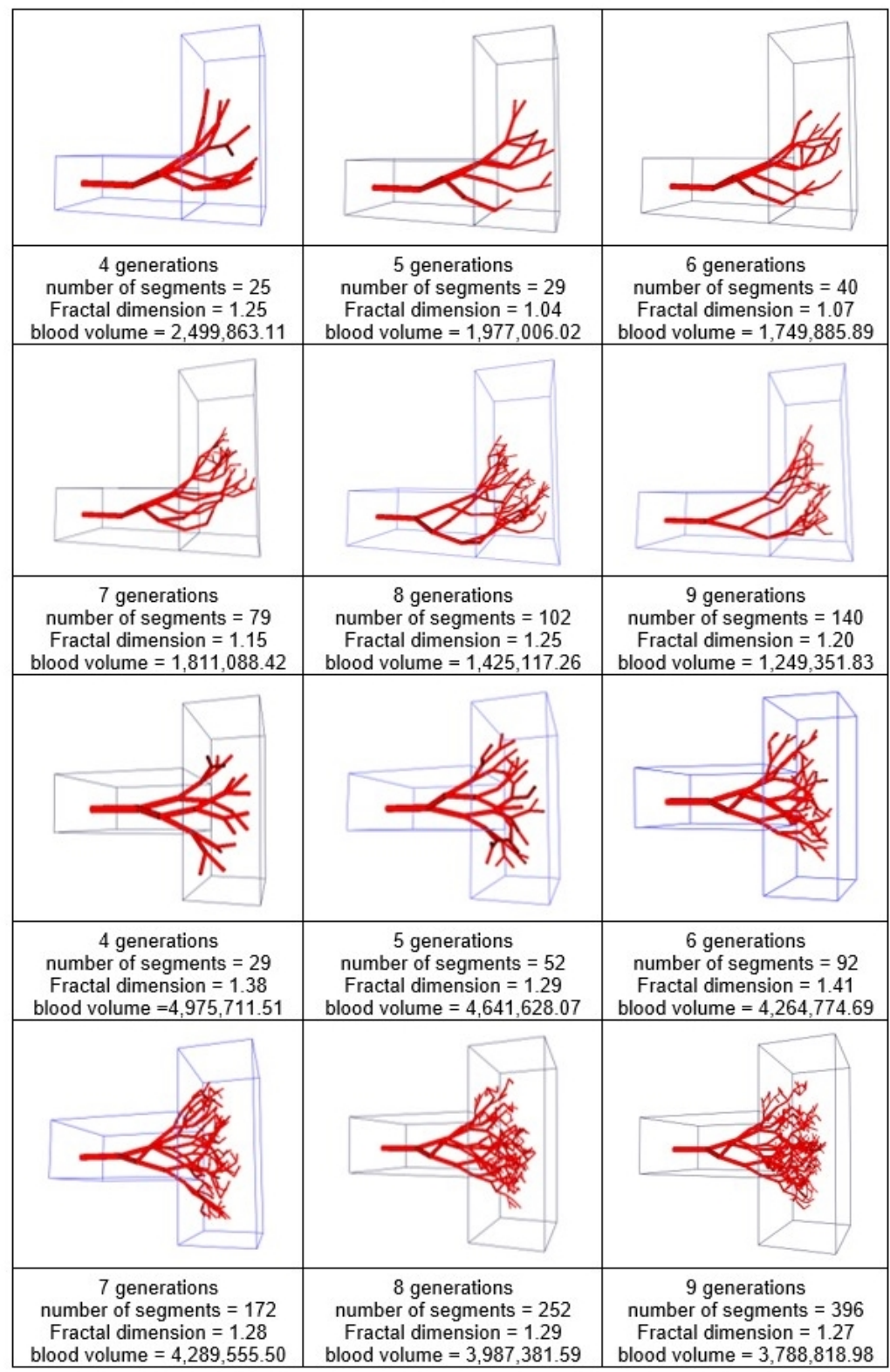

Figure 8 Structures of blood vessels generated in $\mathrm{L}$ and $\mathrm{T}$ domains in different generations.

\section{Discussion}

As previously discussed in the previous section, the construction model and algorithm described in section 2 can be used to simulate structures of blood vessels in different sceneries and domains. To illustrate how the results of 3D structures can be used or applied for future studies, we compare with other results appeared in literature.

Figure 9 shows comparisons of structures of blood vessels simulated in this work with other simulations in literature. Figures 9(a) and 9(b) shows the structures of blood vessels constructed from the model, and compared with a similar image appeared in the work of Tena and Clara [32] (Figure 9(c)) for 
branching tree structure in lungs for the study of aerosol deposition in lung. This suggests that constructed structures in this work could be also used for study of flow in lung, which could be done algorithmically via L-system code file.

Figures 9(d) and 9(e) shows the structures of blood vessels constructed in this work compared with the simulation of structure of blood vessels generated from micro-CT images of rat renal vasculature in the work of Nordsletten et al. [9] (Figure 9(f)). This comparison suggests that our constructed structures could be also used as a model to study property of blood flows in Nordsletten et al. [9].

Figures 9(g) and 9(h) shows the structures of blood vessels constructed in this work compared with the simulation structure by Yang and Wang [3] (Figure 9(i)). In their work, they constructed 3D structures of blood vessels based on the power law with the lengths of blood vessels controlled by Gaussian distribution; however, the simulations were generated without using L-system.

From these comparisons, we see that the construction model and algorithm developed in this work can produce structures of blood vessels similar to other existing structures appeared in literature. Thus, the obtained results could be used and applied for further investigation some important property of structures of blood vessels, such as topics in blood flow [26], pressure [27] and coronary artery disease [28]. The possible advantage of this work relies on the fact that structures of blood vessels are coded as Lsystem file, which would be easier to modify and implement than using images of structures.

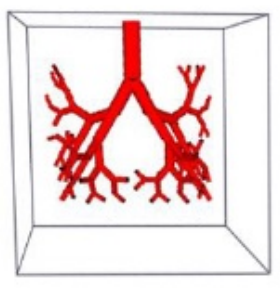

(a)

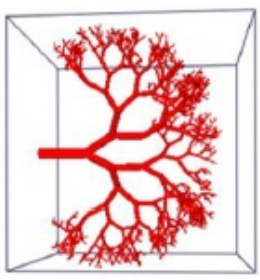

(d)

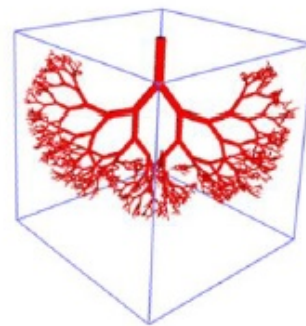

(g)

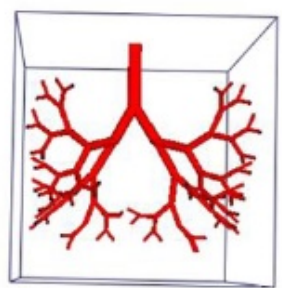

(b)

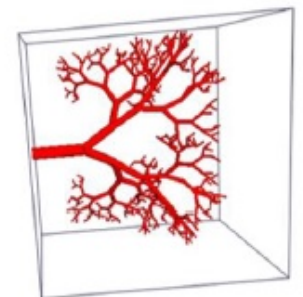

(e)

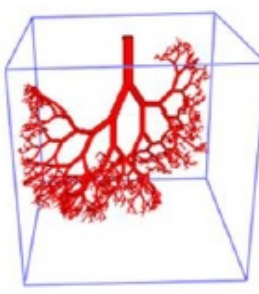

(h)

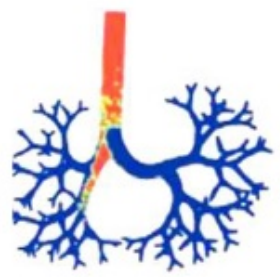

(c)

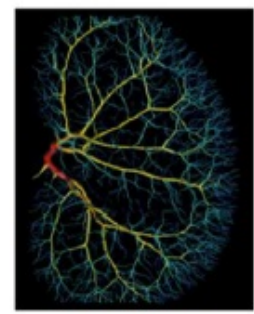

(f)

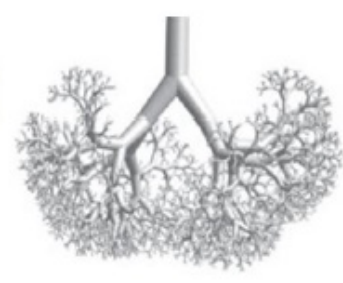

(i)

Figure 9 Comparison of simulated structures of blood vessels in organs. In lung: (a) and (b) constructed structures vs. (c) image in Tena and Clara [32]; in kidney: (d) and (e) constructed structures vs. (f) simulation in Nordsletten et al. [9]; and (g) and (h) constructed structures vs. (i) structure simulated by Yang and Wang [3]. 


\section{Conclusions}

In this work we have developed construction model and algorithm for simulating structures of blood vessels. The construction relies on our modified 3D L-system and on the physiological law of arterial branching proposed Murray (Murray's law) with additional assumptions and constraints such as the spreading of blood vessels to cover all directions, the angle condition, and the non-overlapping vessels condition. We have also created a software based on openGL and Lazarus program to visualize and generate L-system codes of structures of blood vessels. The construction model is applied to simulate structures of blood vessels in 3 study cases: 1) structures with symmetric arterial branching, 2) structures with non-symmetric arterial branching and 3) structures of blood vessels for $\mathrm{L}$ and $\mathrm{T}$ shape domains. The validation of construction model is illustrated by comparing the simulated structures of blood vessels with other existing results in literature. This concludes that the model developed in this work for constructing structures of blood vessels could be used as basic structures for other study topics such as blood flow, blood pressure and some properties of structures of blood vessels. The advantage of this work also relies on the L-system code files which store information of blood vessel structures; namely, 1) the L-system code requires less space than storing using images and 2) modification on L-system file is easier to perform than using images when investigating properties of blood vessel structures such as blood flow and pressure on different organs.

\section{Acknowledgments}

This research is supported from the Ministry of Science and Technology (Thailand).

\section{References}

[1] CD Murray. The physiological principle of minimum work applied to the angle of branching of arteries. J. Gen. Physiol. 1926; 9, 835-41.

[2] E Fanucci, A Orlacchio and M Pocek. The vascular geometry of human arterial bifurcations. Invest. Radiol. 1988; 23, 713-8.

[3] J Yang and Y Wang. Design of vascular networks: A mathematical model approach. Int. J. Numer. Methods Biomed. Eng. 2013; 29, 515-29.

[4] M Zamir. Nonsymmetrical bifurcation in arterial branching. J. Gen. Physiol. 1978; 72, 837-45.

[5] M Zamir, JA Medeiros and TK Cunningham. Arterial bifurcation in the human retina. J. Gen. Physiol. 1979; 74, 537-48.

[6] M Marxen and RM Henkelman. Branching tree model with fractal vascular resistance explains fractal perfusion heterogeneity. Am. J. Physiol. Heart Circ. Physiol. 2003; 284, H1848-H1857.

[7] M Zamir. Distributing and delivering vessels of the human heart. J. Gen. Physiol. 1988; 91, 725-35.

[8] MH Tawhai, AR Clarka, GM Donovan and KS Burrowes. Computational modeling of airway and pulmonary vascular structure and function: Development of a "lung physiome". Crit. Rev. Biomed. Eng. 2011; 39, 319-36.

[9] DA Nordsletten, S Blackett, MD Bentley, EL Ritman and NP Smith. Structural morphology of renal vasculature. Am. J. Physiol. Heart Circ. Physiol. 2006; 291, 296-309.

[10] M Zamir, SM Wrigley and BL Langille. Arterial bifurcations in the cardiovascular system of a rat. J. Gen. Physiol. 1983; 81, 325-35.

[11] W Schreiner, F Neumann, M Neumann, A End, SM Roedler and S Aharinejad. The influence of optimization target selection on the structure of arterial tree models generated by constrained constructive optimization. J. Gen. Physiol. 1995; 106, 583-99.

[12] W Schreiner, F Neumann, M Neumann, R Karch, A End and SM Roedler. Limited bifurcation asymmetry in coronary arterial tree models generated by constrained constructive optimization. $J$. Gen. Physiol. 1997; 109, 129-40.

[13] W Schreiner, R Karch, M Neumann, F Neumann, P Szawlowski and S Roedler. Optimized arterial trees supplying hollow organs. Med. Eng. Phys. 2006; 28, 416-29.

[14] R Karch, F Neumann, M Neumann and W Schreiner. Functional characteristics of optimized arterial tree models perfusing volumes of different thickness and shape. J. Vasc. Res. 2000; 37, 250-64.

[15] W Schreiner, M Neumann, F Neumann, SM Roedler, A End, P Buxbaum, MR Muller and P Spieckermann. The branching angles in computer-generated optimized models of arterial trees. $J$. Gen. Physiol. 1994; 103, 975-89.

[16] E Fanucci, A Orlacchio, M Pocek, A Magrini and E Salomoni. Optimal branching of human arterial bifurcations. Invest. Radiol. 1990; 25, 62-6. 
[17] R Karch, F Neumann, BK Podesser, M Neumann, P Szawlowski and W Schreiner. Fractal properties of perfusion heterogeneity in optimized arterial trees: A model study. Invest. Radiol. 2003; 122, 307-21.

[18] M Zamir. Arterial branching within the confines of fractal L-system formalism. J. Gen. Physiol. 2001; 118, 267-75.

[19] MA Galarreta-Valverde, MMG Macedo, C Mekkaoui and MP Jackowski. Three-dimensional synthetic blood vessel generation using stochastic L-systems. In: Proceedings of the International Society for Optical Engineering, Florida. 2013, p. 1-6.

[20] M Zamir and N Brown. Arterial branching in various parts of the cardiovascular system. Am. J. Anat. 1982; 163, 295-307.

[21] P Prusinkiewicz. Graphical applications of L-systems. In: Proceedings of the Graphics Interface ' 86 - Vision Interface '86, Vancouver, British Columbia, Canada. 1986, p. 247-53.

[22] P Prusinkiewicz and A Lindenmayer. The algorithmic beauty of plants. Springer-Verlag New York, New York, 1990, p. 1-50.

[23] P Prusinkiewicz, J Hanan, M Hammel and R Mech. L-systems: From the theory to visual models of plants. In: Proceedings of the $2^{\text {nd }}$ CSIRO Symposium on Computational Challenges in Life Sciences, Melbourne, Australia. 1996, p. 1-27.

[24] K Guan. The research of the improved 3D L-system and its application in plant modeling. In: Proceedings of the IEEE Conference on Cybernetics and Intelligent Systems, Chengdu, China. 2008, p. 718-23.

[25] G Gutierrez, HD Reines and ME Wulf-Gutierrez. Clinical review: Hemorrhagic shock. Crit. Care 2004; 8, 373-81.

[26] LO Schwenl and T Preusser. Analysis and algorithmic generation of hepatic vascular systems. Int. J. Hepatol. 2012; 2012, 357687.

[27] PJ Blanco, LO Muller and JD Spence. Blood pressure gradients in cerebral arteries: A clue to pathogenesis of cerebral small vessel disease. Stroke Vasc. Neurol. 2017; 2, 108-17.

[28] A Gholipour, M Ghayesh and A Zander. Nonlinear biomechanics of bifurcated atherosclerotic coronary arteries. Int. J. Eng. Sci. 2018; 133, 60-83.

[29] M Dong, W Yang, JS Tamaresis, FP Chan, EJ Zucker, S Kumar, M Rabinovitch, AL Marsden and JA Feinstein. Image-based scaling laws for somatic growth and pulmonary artery morphometry from infancy to adulthood. Am. J. Physiol. Heart Circ. Physiol. 2020; 319, 432-42.

[30] ID Kelch, G Bogle, GB Sands, ARJ Phillips, IJ LeGrice and PR Dunbar. Organ-wide 3D-imaging and topological analysis of the continuous microvascular network in a murine lymph node. Sci. Rep. 2015; 5, 16534.

[31] PJ Blanco, RABD Queiroz and RA Feijoo. A computational approach to generate concurrent arterial networks in vascular territories. Int. J. Numer. Methods Biomed. Eng. 2013; 29, 601-14.

[32] AF Tena and PC Clara. Deposition of inhaled particles in the lungs. Arc. Bronconeumol. 2012; 48, 240-6. 